\title{
Entwicklung einer ergonomischen Lagerplatzvergabe in der manuellen Person-zur-Ware Kommissionierung
}

\author{
Tim Steinebach ${ }^{1}$ Jurij Wakula' $\cdot$ Ralph Bruder ${ }^{1}$ Maximilian Paulke'
}

Angenommen: 17. Juni 2021 / Online publiziert: 9. Juli 2021

(c) Der/die Autor(en) 2021

\section{Zusammenfassung}

In diesem Beitrag soll eine ergonomische Lagerplatzvergabe für Lager mit Fachbodenregalen entwickelt werden, welche Artikel bestimmten Lagerplätzen entsprechend der benötigten Kommissionierzeit und der körperlichen Belastung optimal zuordnet. Zur Bestimmung der Zeit wird der MTM-UAS Standard genutzt. Da Erkrankungen im unteren Rückenbereich bei Kommissionierenden besonders häufig auftreten, wird als Belastungskenngröße die Druckkraft auf das Wirbelsäulensegment L5-S1 gewählt. Zur Ermittlung dieser Kenngröße werden Kommissionierversuche $(n=11)$ durchgeführt und eingenommene Körperhaltungen in ein biomechanisches Simulationsprogramm implementiert. Die Zeit- und Belastungskenngrößen werden in einer bikriteriellen Optimierung pareto-effizient gelöst, wobei aufgrund des Zielkonflikts Kompromisslösungen der beiden Zielgrößen besonders attraktiv sind.

Praktische Relevanz: In der Kommissionierung ist der Anteil von manuellen Tätigkeiten hoch, sodass Mitarbeitende potenziell hohen körperlichen Belastungen ausgesetzt sind. Planungskonzepte in der Kommissionierung, die ergonomische Kriterien berücksichtigen, sind selten - dieser Ansatz soll entsprechend eine Reduzierung der körperlichen Belastung durch die Lagerplatzvergabe (LPV) ermöglichen. Interessant für praktische Anwender ist, dass die entwickelte LPV unabhängig vom Lagerlayout angewendet werden kann. Im Gegensatz zu anderen Lösungen, wie z.B. Teilautomatisierungen, mechanische Hebehilfen oder Exoskelette, erfordert eine Änderung der LPV zudem kaum Investitionskosten.

Schlüsselwörter Kommissionierung · Lagerplatzvergabe $\cdot$ Biomechanische Belastung $\cdot$ Bikriterielle Optimierung · 3DSSPP

\section{Development of an ergonomic storage location assignment in manual order picking}

\begin{abstract}
In this paper, an ergonomic storage location assignment for order picking warehouses with shelving racks is derived, which optimally assigns the items to be stored to specific storage locations according to the required picking time and the physical workload. The MTM-UAS standard is used to determine the picking time. Since lower back pain is particularly common among order pickers, the compression force on the spinal segment L5-S1 is selected. In order to derive the spinal load, picking experiments $(n=11)$ are carried out. The postures adopted are implemented in a biomechanical simulation programme in order to determine the corresponding compression forces. The time and spinal load parameters are combined in a bi-objective optimisation and solved pareto-efficient. There is a conflict of objectives (time and spinal load)—compromise solutions prove to be particularly attractive.
\end{abstract}

Tim Steinebach, M.Sc.

tim.steinebach@tu-darmstadt.de

1 Institut für Arbeitswissenschaft, Technische Universität

Darmstadt, Otto-Berndt-Straße 2, 64287 Darmstadt,

Deutschland 
Practical Relevance: In order picking, the share of manual activities is high, so that employees are potentially exposed to high physical workload. Planning concepts in order picking that take ergonomic criteria into account are rare-thus, this approach is intended to present a reduction of physical workload through storage location assignment (SLA). Interesting for practical users is that the developed SLA can be applied to item portfolios independently of the warehouse layout. In contrast to other solutions, such as partial automation, mechanical lifting aids or exoskeletons, a change in SLA requires hardly any investment costs, making it easier to implement in practice.

Keywords Order picking $\cdot$ Storage location assignment $\cdot$ Biomechanical load $\cdot$ Bi-objective optimization $\cdot$ 3DSSPP

\section{Einleitung}

Aufgrund des zunehmenden Warenhandels und steigender Kundenanforderungen, wie z.B. die Einhaltung sehr kurzer Lieferzeiten, nimmt das effiziente und nachhaltige Management von Lagerhäusern eine zentrale Rolle in Bezug auf den Unternehmenserfolg ein (ten Hompel et al. 2011). Insbesondere die Gestaltung des Kommissioniersystems hat einen entscheidenden Einfluss auf die Erfüllung von Lieferfristen und damit auf die Kundenzufriedenheit (Grosse et al. 2015).

Hierbei versteht man unter der Kommissionierung das Zusammenstellen von Gütern entsprechend spezifischer Kundenbestellungen aus einem Gesamtsortiment (VDI 3590). Dieser Prozess wird häufig als die komplexeste Aufgabe der innerbetrieblichen Logistik bezeichnet (Gudehus 2012).

Trotz der voranschreitenden Automatisierung zeichnet sich die Intralogistik, z. B. im Vergleich mit der Produktion, nach wie vor durch einen großen Anteil an manuellen Tätigkeiten aus (Grosse et al. 2017). Begründet werden kann dies durch die hohen kognitiven und motorischen Fähigkeiten des Menschen, die eine höhere Flexibilität beim Greifen von Artikeln bietet, als vergleichbare automatisierte Systeme, wie beispielsweise Pickroboter. So geht man davon aus, dass in etwa $80 \%$ der Lagerhäuser vorwiegend manuell nach dem Person-zur-Ware Prinzip kommissioniert wird (Napolitano 2012; Michel 2017). Aufgrund des hohen Personaleinsatzes sind bis zu $50 \%$ der in der Intralogistik entstehenden Kosten alleine auf die Kommissionierung zurückzuführen (Tompkins et al. 2010), was die Bedeutung der effizienten Gestaltung von Kommissioniersystemen verdeutlicht.

Der hohe Anteil manueller Tätigkeiten setzt die Mitarbeitenden zudem hohen physischen Belastungen aus und geht mit einem hohen Risiko zur Verursachung muskuloskelettaler Erkrankungen einher. Das Heben bzw. Umsetzen von hohen Lastgewichten in Kombination mit teilweise ungünstigen Körperhaltungen hat vor allem Auswirkungen auf die Belastung der Lendenwirbelsäule (Jäger et al. 2002). Hierbei können die mechanischen Belastungen sowohl bei kurzzeitigen Vorgängen als auch bei Expositionen über einen langen Zeitraum zu bandscheibenbedingten
Erkrankungen führen (Jäger et al. 2007). Entsprechend ist „lower back pain“ die häufigste Ursache für Gesundheitsbeschwerden in der Kommissionierung (Kretschmer et al. 2021) und kommt Studien zufolge nur bei $6 \%$ aller Kommissionierenden nie vor (Gajšek et al. 2020).

Einen entsprechend hohen Stellenwert sollte neben der Steigerung der Wirtschaftlichkeit von Lagern die Prävention von Gesundheitsbeschwerden bzw. die Reduzierung der Wirbelsäulenbelastung besitzen. Jedoch sehen über $85 \%$ der Fachleute aus der Intralogistik noch zumindest mittleren bis sehr großen Bedarf zur Verbesserung der Ergonomie an entsprechenden Arbeitsplätzen (Spath et al. 2019). Insbesondere existieren bislang nur sehr wenige Ansätze, bei denen ergonomische Kriterien in den klassischen Planungskonzepten der Kommissionierung, wie z. B. der Lagerplatzvergabe, der Routenführung oder der Layoutplanung berücksichtigt werden (Grosse et al. 2015, 2017). Vor allem die Lagerplatzvergabe hat das Potenzial ohne hohe Investitionen die Belastung durch die manuelle Lastenhandhabung direkt zu beeinflussen, da sie darüber entscheidet, welche Lastgewichte aus welchen Entnahmehöhen von den Kommissionierenden wie oft gepickt werden müssen (Battini et al. 2016; Larco et al. 2017).

Ziel dieses Beitrags ist es daher, eine bikriterielle Optimierung der Lagerplatzvergabe für eine Lagerung in Regalen vorzunehmen, welche sowohl die Durchlaufzeit der Kommissionierung als auch die Lumbalbelastung berücksichtigt. Als Belastungsparameter soll dabei die Druckkraft auf die Bandscheibe L5-S1 dienen.

\section{Literaturüberblick}

\subsection{Ergonomische Lagerplatzvergabe}

Die Lagerplatzvergabe (LPV) bezeichnet die Zuordnung von Artikeln zu örtlich definierten Lagerplätzen (Bernnat 1997). Grundsätzlich unterscheidet man zwischen zufälliger, klassenbasierter und fester LPV (de Koster et al. 2007). Während die zufällige Lagerplatzvergabe zu kommissionierende Objekte (stock keeping units; SKU) zufällig im Lager zuweist und somit eine höhere Auslastung der Lagerkapazität aufweist (da z. B. für nicht vorrätige Artikeln kein Platz 
freigehalten wird), können bei Festplatzprinzipien SKU entsprechend ihrer Eigenschaften zugewiesen werden, um eine effizientere Kommissionierung zu ermöglichen. Klassische Eigenschaften, anhand derer die Entscheidung über einen bestimmten Lagerplatz abgeleitet werden, sind die Zugriffshäufigkeit, etwaige Zugriffskorrelationen, das Volumen (Cube Order Index) oder seltener das Lastgewicht. Die klassenbasierte LPV ist eine Mischform und ordnet Artikel entsprechend ausgewählter Eigenschaften bestimmten Zonen im Lager zu, in denen wiederum zufällige Lagerplätze ausgewählt werden (Qin et al. 2015).

Reyes et al. (2019) konnten in ihrem Literaturüberblick zeigen, dass für die große Mehrheit an Ansätzen zur Lagerplatzvergabe rein ökonomische Kennzahlen, wie z.B. die im Lager zurückgelegte Wegstrecke oder die daraus abgeleiteten Kosten, verwendet werden.

$\mathrm{Zu}$ den ersten Autoren, die menschliche Eigenschaften in ihre Optimierungsmodelle einbezogen gehörten Petersen et al. (2005). In ihrem Modell konnte nachgewiesen werden, dass eine Platzierung von häufig gegriffenen SKU in der „Goldenen Zone“ (Entnahmehöhe zwischen Schulter und Hüfte) zu geringeren Durchlaufzeiten führt, auch wenn hierdurch längere Distanzen zurückgelegt werden müssen. Physische Belastungen bzw. Beanspruchungen wurden jedoch nicht berücksichtigt.

Die wenigen Ansätze, die physische Belastungsparameter in der Modellentwicklung aufnehmen, basieren meist auf dem Energy Expenditure Modell (Garg et al. 1978) und berechnen den aus Einzeltätigkeiten additiv resultierenden Energieumsatz (Battini et al. 2016; Calzavara et al. 2019). So kann nach Battini et al. (2016) in einem Lager mit einer Regalreihe neben der zeitlichen Komponente auch die energetische Belastung minimiert und pareto-effiziente Lösungen gefunden werden, bei denen eine der beiden Zielgrößen nicht verbessert werden kann, ohne die jeweils andere zu verschlechtern. Diefenbach und Glock (2019) greifen ebenfalls auf das Energy Expenditure Modell nach Garg et al. (1978) zurück, um für u-förmige Kommissionierzonen sowohl Designempfehlungen für das Layout als auch für die Lagerplatzvergabe abzuleiten. Calzavara et al. 2019 wenden zusätzlich zum Energieumsatz das OWAS-Verfahren (Karhu et al. 1977) an, um den Einfluss verschiedener Entnahmepositionen auf einer Palette bezüglich der Körperhaltung in das Modell zu integrieren.

Ferner wurde die Borg CR-10 Skala (Borg 1982) von Larco et al. (2017) genutzt, um mittels Regressionsanalysen die Einflussgrößen Entnahmehöhe, Gewicht und $\mathrm{Zu}$ griffshäufigkeit auf die subjektiv wahrgenommene Beanspruchung der Kommissionierenden zu ermitteln. Die subjektive Beanspruchung wurde ähnlich wie in Battini et al. 2016 in einer bikriterielle Optimierung genutzt, um paretoeffiziente Zuordnungen von SKU zu Lagerplätzen zu ermitteln.
Die ermittelten Werte des Energieumsatzes bzw. die subjektiven Beanspruchungswerte werden in den genannten Veröffentlichungen jedoch nicht explizit angegeben bzw. hergeleitet und können deshalb nur schwer auf andere Lagerlayouts übertragen werden. Zudem wurde beim Einfluss des Lastgewichts auf die subjektiv empfundene Beanspruchung nur zwischen leichten $(\leq 3 \mathrm{~kg})$ und schweren $(>3 \mathrm{~kg})$ Artikeln unterschieden.

Einen Ansatz, der auch biomechanische Kriterien berücksichtigt, stammt von Otto et al. (2017). Mit der NIOSH Revised Lifting Equation (Waters et al. 1994) werden Entnahmevorgängen von SKU aus bestimmten Entnahmehöhen Belastungskennwerte zugewiesen. Diese erlauben eine Minimierung der Gesamtbelastung durch eine Lagerplatzzuweisung und eine Einteilung von Mitarbeitenden zu bestimmten Lagerzonen. Mittels Computersimulation konnte gezeigt werden, dass eine Vernachlässigung ergonomischer Kriterien zu deutlich erhöhten physischen Belastungen führen kann - eine gleichzeitige Betrachtung der Auswirkungen auf zeitliche Parameter wurde jedoch nicht durchgeführt.

Obwohl Lower Back Pain die häufigste Ursache für Gesundheitsbeschwerden in der Kommissionierung ist (Gajsek et al. 2020; Kretschmer et al. 2021), konnten in der Literatur keine Beiträge zur Lagerplatzvergabe gefunden werden, welche direkt die Belastung der Wirbelsäule berücksichtigen. Zudem sind verwendete Verfahren wie die NIOSH Lifting Equation, welche teilweise auf biomechanischen Untersuchungen beruht, nur bedingt in der manuellen Kommissionierung geeignet, da diese lediglich für langsam und gleichmäßig ausgeführte Hebevorgänge bei konstanten Lastgewichten konzipiert wurde (Waters et al. 1994; Goldscheid 2008). Ferner handelt es sich bei den genannten Ansätzen lediglich um mathematische Modelle, die nicht empirisch evaluiert wurden.

\subsection{Lumbalbelastung in der Kommissionierung}

Da im Rahmen der zu entwickelnden LPV die Lumbalbelastung berücksichtigt werden soll, werden im folgenden wissenschaftliche Untersuchungen in der manuellen Kommissionierung vorgestellt, die biomechanische Modelle verwendeten, um Kompressions- bzw. Scherkräfte auf die unteren beiden Bandscheiben der Lendenwirbelsäule L4-L5 bzw. L5-S1 per Simulation ermittelten, da diese besonders häufig Schädigungen aufweisen (Jäger et al. 2002). Dabei muss zwischen einer kurzzeitigen und einer kumulativen Exposition unterschieden werden. Während im NIOSHVerfahren einheitlich eine maximale Kompressionskraft von $3,4 \mathrm{kN}$ auf die lumbosakrale Bandscheibe zugrunde gelegt wird (Waters et al. 1994), differenzieren die Revidierten Dortmunder Richtwerten zwischen verschiedenen Altersklassen und dem Geschlecht. Die empfohlenen ma- 
ximalen Kompressionskräfte beim Handhaben von Lasten liegen bei jüngeren bzw. älteren Männern im Bereich zwischen 5,4 und 2,2 kN sowie bei jüngeren und älteren Frauen zwischen 4,1 und 1,8 kN (DGUV 2020).

Eines der detailliertesten Modelle zur Bestimmung von mechanischen Belastungen der Wirbelsäule ist „,der Dortmunder", welcher die Simulation von asymmetrischen und dynamischen (Massenträgheit) Tätigkeiten ermöglicht (Jäger et al. 2000). So wurden Tabellen für die Maxima von Druck- und Scherkräften auf die Bandscheibe L5-S1 mit bestimmten Gewichten und verschiedenen manuellen Handhabungsvorgängen, wie z. B. das Heben und Umsetzen (Jäger et al. 2002) oder das Tragen (Jordan et al. 2001) von Lasten, erstellt. Auf Basis dieser Ergebnisse entwickelte Goldscheid (2008) eine Systematik zur Erfassung der Wirbelsäulenbelastung in der manuellen Kommissionierung.

Zudem wurden in der Forschung bezüglich Problemstellungen in der Lagerhaltung bzw. Kommissionierung auch weniger komplexe, statische Modelle genutzt, wie z. B. Jack Computer Manikin oder 3DSSPP.

Hanson et al. (2018) untersuchten mit dem Jack Computer Manikin, inwiefern sich die benötigte die Kompressionskraft auf die L4-L5 Bandscheibe bei Picking-Aktivitäten aus großen Containern durch eine Neigung um $45^{\circ}$ verändern. Ebenso analysierten Ulin und Keyserling (2004) die Auswirkung der Neigung einer Palette sowie die Höhenverstellbarkeit eines Kommissionierwagens auf die Lumbalbelastung mittels dem Simulationsprogramm 3DSSPP. Beide Maßnahmen konnten die Zeit, die in ungünstigen Körperhaltungen verbracht wird und damit die mechanische Belastung der Wirbelsäule signifikant reduzieren. Ferner nutzten Glock et al. (2019) das zweidimensionale 4D WATBAK, um Vorteile rotierbarer Paletten zu untersuchen. Beim Kommissionieren vom hinteren Teil der Palette begünstigt das Rotieren aufgrund der geringeren horizontalen Abstände die eingenommenen Körperhaltungen und reduziert somit nicht nur die Kompressionskräfte auf die Bandscheibe L4-L5, sondern auch die benötigte Kommissionierzeit.

\subsection{Systeme vorbestimmter Zeiten: MTM-Verfahren}

Mit Hilfe der Zeitwirtschaft werden Zeitdaten für einzelne Arbeitsschritte ermittelt, die zur Planung, Steuerung und Kontrolle von Arbeitsprozessen genutzt werden, um einen optimalen Ressourceneinsatzes gewährleisten zu können (Schlick et al. 2018). Um die benötigten Zeitdaten $\mathrm{zu}$ ermitteln, existieren verschiedene Methoden der Zeitwirtschaft, die sich in analytisch-experimentelle sowie analytisch-rechnerische Methoden aufteilen (VDI 4481). Während die analytisch-experimentellen Methoden real vorliegende Arbeitssysteme voraussetzen und deshalb für Planungszwecke nicht geeignet sind, bieten sich für diesen
Beitrag vor allem analytisch-rechnerische Methoden an. Nennenswert sind insbesondere jene Verfahren, die Zeiten durch das Zusammensetzen von Zeitbausteinen für manuelle (Teil-)Arbeitsvorgänge ermitteln. Diese ergeben sich aus Bewegungsanalysen, die auch Systeme vorbestimmter Zeiten (SvZ) genannt werden. Weltweit etabliert hat sich hierbei vor allem das Verfahren „Methods-Time Measurement" (MTM), welches entlang des gesamten PEPProzesses in verschiedenen Branchen angewendet wird (Bokranz und Landau 2012).

Die Grundlage aller MTM-Prozessbausteinsysteme stellt das Grundverfahren MTM-1 dar. Darauf aufbauend sind verdichtete Prozessbausteinsysteme entwickelt worden, die sich in ihrem Methodenniveau unterscheiden, welches angibt, wie groß die ,einen Prozesstyp repräsentierende Routinebildungschance“" ist (Bokranz und Landau 2012). Je höher das Methodenniveau ist, desto genauer muss der Ablauf des Prozesses aufgezeigt werden. MTM-Prozessbausteinsysteme, mit einem geringeren Methodenniveau als MTM-1 sind beispielsweise das Universelle Analysiersystem (MTM-UAS) oder das MTM in der Einzel- und Kleinserienfertigung (MTM-MEK) zu nennen. Für alle MTM-Prozessbausteinsysteme existieren MTM-Datenkarten. Diese enthalten Normzeitwerte von eingearbeiteten, durchschnittlich veranlagten Mitarbeitern für die in Abhängigkeit der jeweiligen Ablaufkomplexität aufgegliederten Bewegungen (Stinson und Wehking 2012). Die Normzeit wird in der Zeiteinheit Time Measurement Unit (TMU) angegeben (Schlick et al. 2018). Weiterführende Literatur zu dem Thema MTM in der Kommissionierung ist in Galka und Günthner (2016) sowie Walch et al. (2009) zu finden.

\section{Modellbildung}

Die hier zu entwickelnde ergonomische LPV soll zwei Zielgrößen berücksichtigen - die Lumbalbelastung (hier: Kompressionskraft auf Bandscheibe L5-S1) und die Systemleistung (hier: benötigte Kommissionierzeit). Der Zusammenhang soll mathematisch beschrieben werden und sich aus Kennzahlen beider Zielgrößen zusammensetzen. Das entsprechende Arbeitsmodell wird in Abb. 1 mitsamt den verschiedenen Einflussfaktoren dargestellt.

Anzumerken ist, dass das Modell in diesem ersten Ansatz auf die wichtigsten Einflussfaktoren beschränkt wird, um die beiden Zielgrößen sinnvoll in einem Optimierungsmodell verbinden zu können. So werden Einflussgrößen, wie beispielsweise die Massenträgheit der Artikel oder menschliche Fähigkeiten oder Fertigkeiten nicht berücksichtigt bzw. konstant gesetzt, um die Komplexität des Modells zu begrenzen und somit die Lösbarkeit des Optimierungsproblems zu gewährleisten. 
Abb. 1 Modell zur Integration der beiden Zielgrößen in einer ergonomischen LPV

Fig. 1 Model for the integration of the two target variables in an ergonomic storage space allocation system

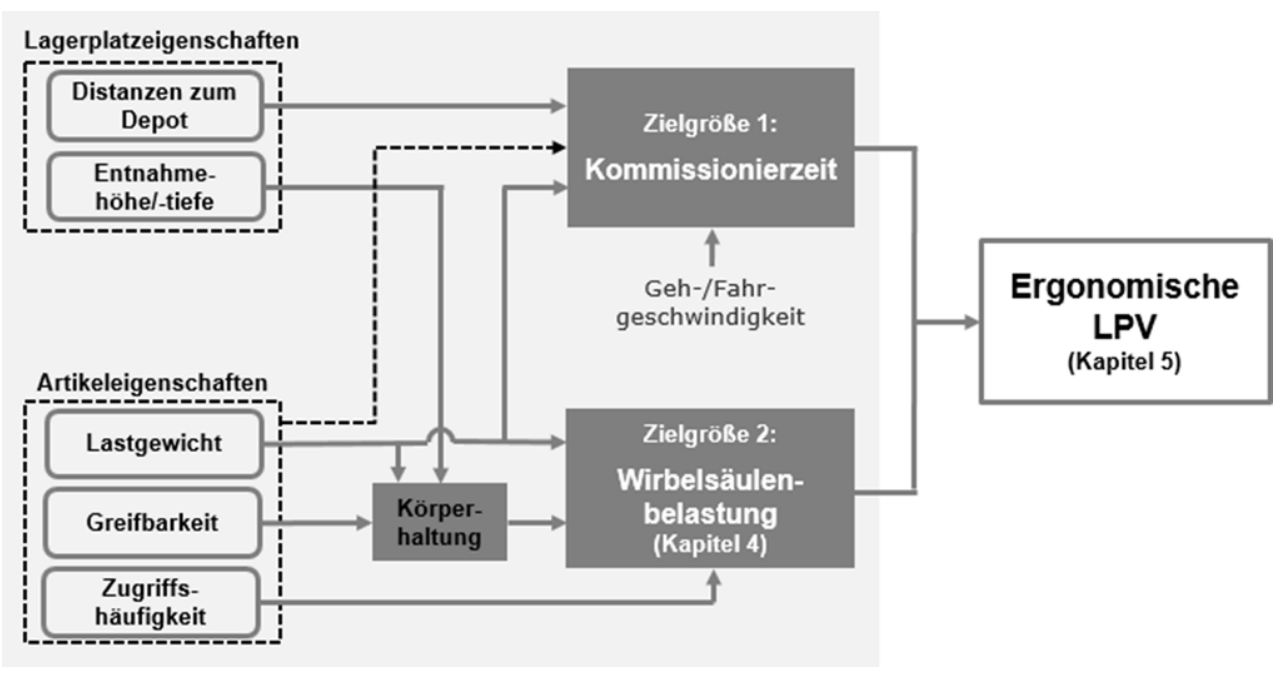

Zunächst soll die Kommissionierzeit betrachtet werden. Diese setzt sich aus der Basiszeit, der Wegzeit, der Greifzeit und der Totzeit zusammen (VDI 4481). Während die Basiszeit und Totzeit (Suchen und Identifizieren von Artikeln) als konstante Zeitwerte angenommen werden können, sind die Wegzeit und die Greifzeit direkt von dem jeweiligen Lagerplatz abhängig. Der größte Anteil der Kommissionierzeit wird in der Regel durch die Wegzeit verursacht (Galka und Günthner 2016), welche direkt von der Distanz des Lagerplatzes zum Depot, also der horizontalen Komponente des Lagerplatzes, sowie von der Geh- bzw. Fahrgeschwindigkeit abhängt. Beim Einsatz von Kommissionierfahrzeugen sind Geschwindigkeiten von $2,5 \mathrm{~m} / \mathrm{s}$ möglich, während beim manuellen Schieben eines Kommissionierwagens durchschnittlich $0,75 \mathrm{~m} / \mathrm{s}$ angenommen werden (Gue et al. 2006; Elbert et al. 2015).

Die Zeit zum Greifen eines SKU hingegen wird neben den verschiedenen Artikeleigenschaften, wie dem Lastgewicht oder der Greifbarkeit (z. B. Griffgestaltung oder Volumen/Sperrigkeit) maßgeblich von der vertikalen Komponente des Lagerplatzes beeinflusst. So wird für eine Entnahme auf Hüft- oder Ellenbogenhöhe weniger Zeit benötigt, als wenn sich die Kommissionierenden tief bücken oder hoch strecken müssen (Galka und Günthner 2016; Petersen et al. 2005).

Nach Galka und Günthner (2016) gibt es zwei Möglichkeiten die Greifzeit in der manuellen Kommissionierung zu bestimmen. Zum einen mithilfe der von Gudehus (2012) entwickelten halbempirische Entnahmezeitformel für Kommissioniertätigkeiten, welche jedoch wenig etabliert und in ihrem Anwendungsbereich stark limitiert ist. Zum anderen können Systeme vorbestimmter Zeiten verwendet werden, wobei für die Kommissionierung besonders das MTM-UAS empfohlen wird. MTM-UAS ermöglicht unter anderem die Nutzung des Vorgangs „Aufnehmen und Platzieren“ und ordnet diesem in Abhängigkeit des Lastgewichts, der Be- wegungslänge und der Schwierigkeit der Artikelaufnahme einen standardisierten Zeitwert zu, siehe Abb. 2. Zudem entwickelte die Deutsche MTM-Vereinigung ein Bausteinsystem „Standardvorgänge Logistik“ (Kuhlang 2014), welches zusätzliche Werte für Tätigkeiten mit Stapler, Hubwagen, Kran (in diesem Beitrag nicht berücksichtigt) sowie Ergänzungswerte für die Handhabung von Lasten bereitstellt. Auf diese Standards soll in diesem Beitrag zurückgegriffen werden.

Die Bestimmung der benötigten Kommissionierzeit eines Artikels aus einem vorgegebenen Lagerplatz kann somit durch die Addition von den zuvor erwähnten Bestandteilen erfolgen. $\mathrm{Zu}$ berücksichtigen ist hierbei insbesondere die Abhängigkeit der Weg- und Greifzeit. Erstere wird durch die Division der Wegstrecke durch die Geh- bzw. Fahrgeschwindigkeit ermittelt. Zur Bestimmung der Wegstrecke ist zu unterscheiden, ob es sich um ein Single-Order bzw. ein Multi-Order Picking handelt, bei denen ein Artikel bzw.

\begin{tabular}{|c|c|c|c|c|c|c|}
\hline \multirow[b]{2}{*}{ 窇 } & \multirow[b]{2}{*}{ 䔍 } & \multirow[b]{2}{*}{ 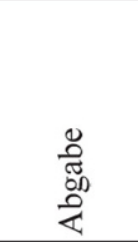 } & \multirow[b]{2}{*}{$\underset{\mathscr{D}}{\tilde{D}}$} & \multicolumn{3}{|c|}{ Bewegungslänge } \\
\hline & & & & 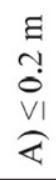 & 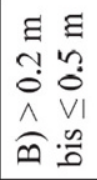 & 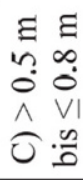 \\
\hline \multirow{3}{*}{$\frac{\stackrel{\infty}{\underline{y}}}{\mathrm{~V}}$} & leicht & ungefähr & $\mathrm{AA}$ & 20 & 35 & 50 \\
\hline & schwierig & ungefähr & $\mathrm{AD}$ & 20 & 45 & 60 \\
\hline & Hand voll & ungefähr & $\mathrm{AG}$ & 40 & 65 & 80 \\
\hline \multicolumn{2}{|c|}{$>1$ bis $\leq 8 \mathrm{~kg}$} & ungefähr & $\mathrm{AH}$ & 25 & 45 & 55 \\
\hline \multicolumn{2}{|c|}{$>8$ bis $\leq 22 \mathrm{~kg}$} & ungefähr & $\mathrm{AL}$ & 80 & 105 & 115 \\
\hline
\end{tabular}

Abb. 2 Datenkarte MTM-AUS (Galka und Günthner 2016)

Fig. 2 MTM-AUS data map (Galka und Günthner 2016) 
mehrere Artikel in einer Tour kommissioniert werden, bevor die Kommissionierenden zum Depot zurückkehren.

Für die Greifzeit wird in diesem Beitrag davon ausgegangen, dass alle Artikel einfach zu greifen und nur ,ungefähr“ platziert werden müssen (siehe Galka und Günthner 2016). Bei einem bekannten Artikeldatensatz lassen sich diese Eigenschaften jedoch im zu entwickelnden Algorithmus problemlos berücksichtigen. Die Lastgewichte der Artikel können direkt in eine der drei Gewichtskategorien eingeordnet werden: Daher sind die entsprechenden Codes AA für leichte Artikel (Lastgewicht $\leq 1 \mathrm{~kg}$ ), AH für mittelschwere Artikel $(>1 \mathrm{~kg}$ und $\leq 8 \mathrm{~kg})$ und AL für schwere Artikel $(>8 \mathrm{~kg})$ auszuwählen. $\mathrm{Ob}$ ein Artikel ein- oder beidhändig gegriffen wird, beeinflusst die Bestimmung der Gewichtsklasse nicht (Bokranz und Landau 2012).

Ebenso ordnet man den einzelnen Lagerplätzen eine bestimmte Bewegungslänge zu. Bei der Bestimmung der Bewegungslängen, die mit einer Entnahme aus den entsprechenden Regalebenen anfallen und als Grundlage für die Einteilung dienen, findet die 8. UAS Regel Anwendung. Diese besagt, dass für die Bestimmung des Entfernungsbereichs die größte zurückgelegte Entfernung maßgebend ist (Bokranz und Landau 2012, S. 487). Da der Start- und Endpunkt des Pickvorgangs nicht identisch sind, ist der Weg nach der Entnahme des SKU maßgebend. Eine Bewegungslänge von unter $20 \mathrm{~cm}$ tritt kaum auf, da die Distanz zwischen Lagerplatz und Kommissionierwagen in der Regel größer ist. Bei niedrigliegenden oder sehr hohen Lagerplätzen ist die Bewegungslänge in Kategorie C) einzuordnen, da bereits die vertikale Bewegungslänge über $50 \mathrm{~cm}$ liegt. Entsprechend sind die einzelnen Bewegungslängen für jeden Lagerplatz zu bestimmen. Bei der Ermittlung der Greifzeit von SKU, die auf den unteren Regalebenen lagern, wird zusätzlich noch ein Prozessbaustein betrachtet, der das ,Senken und Aufrichten des Rumpfes“ durch Beugen, Bücken oder Knien analysiert (Bokranz und Landau 2012). Diese Bewegung wird durch die Angabe der Codierung „,KB“ berücksichtigt Sofern die Kommissionierenden den Kommissionierwagen nicht direkt vor dem Lagerplatz des zu entnehmenden Artikels abstellen und nach der Entnahme noch eine Körperbewegung ausführen, um den Artikel auf dem Kommissionierwagen abzulegen, ist die Analyse des Vorgangs Aufnehmen und Platzieren gemäß der 5. UAS Regel trotzdem zulässig (Bokranz und Landau 2012). Die zwischen Entnahme und Ablage des Artikels auftretende Körperbewegung wird in diesem Fall separat aufgeführt und zeitlich bewertet. In diesem Beitrag wird jedoch angenommen, dass der Kommissionierwagen direkt am Lagerplatz des zu entnehmenden SKU abgestellt wird und zwischen der Entnahme und Ablage des Artikels keine Körperbewegungen anfallen.

Die zweite in der ergonomischen Lagerplatzvergabe betrachtete Zielgröße ist die Lumbalbelastung. Obwohl ener- getische Belastungen z.B. beim Bewegen durch das Lager oder beim Umsetzen von Artikeln durchaus relevant sind, wird sich in diesem Artikel auf die Lumbalbelastung beschränkt, da die am häufigsten vorkommenden Gesundheitsbeschwerden von Lagermitarbeitenden im Lendenwirbelbereich auftreten (Gajšek et al. 2020; Kretschmer et al. 2021).

Bereits in Abschn. 2.2 wird klar, dass die genannten Publikationen keine einheitliche Vorgehensweise zur Ermittlung der Kompressionskräfte aufweisen und in der Vergangenheit mit verschiedenen biomechanischen Modellen softwareunterstützt gerechnet wurde. Zudem sind die veröffentlichten Druckkraftwerte nur bedingt geeignet, um sie als Eingangsparameter für eine ergonomische LPV zu nutzen, da diese nicht für jede Entnahmehöhe bzw. verschiedene Gewichte vorliegen, sodass entsprechende Kenngrößen im nachfolgenden Kap. 4 gezielt abgeleitet werden.

\section{Kommissionierversuche zur Bestimmung von Kennzahlen zur Lumbalbelastung}

\subsection{Untersuchungsmethodik}

Manuelle Lastenhandhabungen sind beim Kommissionieren unumgänglich, weshalb insbesondere die mechanische Belastung der Lendenwirbelsäule bestimmt werden sollte (Jäger et al. 2002). Die im folgenden abgeleiteten Kompressionskräfte auf Bandscheiben der Lendenwirbelsäule sollen für ein Optimierungsverfahren zur LPV in bestehenden Lagern mit Fachbodenregalen verwendet werden. Sehr detaillierte und aufwändige Verfahren, wie z.B. eine biomechanische Simulation mit „dem Dortmunder“ sind nicht zielführend, da diese Verfahren zwar sehr exakte Werte liefern und vielfältige Einflüsse berücksichtigen, die entsprechenden Input-Parameter für das vorliegende Vorhaben jedoch nur mit außerordentlich hohem Aufwand allgemeingültig für alle Versuchsteilnehmenden bestimmt werden können.

Aus diesem Grund wurde sich für das weniger komplexe und allgemein verfügbare 3D Static Strength Prediction Program (3DSSPP) entschieden, welches statische Druckund Scherkräfte der Segmente L4-L5 bzw. L5-S1 durch biomechanische Simulation für verschiedene Lasthandhabungsfälle, wie z. B. Heben und Umsetzen, errechnet. Eine dreidimensionale Körperhaltung kann mittels einem graphischen Menschmodell eingestellt sowie die einwirkende Kraftrichtung und der -betrag festgelegt werden (BullingerHoffmann et al. 2016).

Anzumerken ist, dass sich auf die Lumbalbelastung durch das manuelle Heben bzw. Umsetzen von SKU aus dem Regal auf einen Kommissionierwagen beschränkt und die Belastung durch das Ziehen und Schieben von entsprechenden Wagen vernachlässigt wird. Dies liegt zum einen 
daran, dass mit der LPV die Häufigkeit, mit der z.B. ein Handwagen angeschoben werden muss (Überwindung der „Losreißkräfte“) nicht beeinflusst werden kann. Zum anderen werden in der Kommissionierung zunehmend angetriebene Transportfahrzeuge oder Fördertechniken eingesetzt, sodass ein manuelles Ziehen bzw. Schieben entfällt.

Versuchsaufbau Für die ergonomische LPV werden durchschnittliche Druckkräfte auf die zu untersuchenden Wirbelsäulensegmente verschiedener Arbeitspersonen während manuellen Kommissioniertätigkeiten benötigt, um belastungskritische SKU günstigen Lagerplätzen zuweisen zu können.

Um die eingenommenen Körperhaltungen zu bestimmen, werden in einem Labor Kommissionierversuche durchgeführt, bei denen SKU mit drei unterschiedlichen Lastgewichten $(0,5 \mathrm{~kg} ; 5 \mathrm{~kg} ; 10 \mathrm{~kg}$ - auf höhere Gewichte wurde in den Versuchen verzichtet, um hohe Belastungen $\mathrm{zu}$ vermeiden) aus neun verschieden hohen Regalebenen $(0-160 \mathrm{~cm}$ im Abstand von $20 \mathrm{~cm})$ entnommen wurden. $\mathrm{Zu}$ beachten ist, dass die tatsächliche Greifhöhe etwa $10 \mathrm{~cm}$ über der jeweiligen Regalhöhe liegt.

Die zu handhabenden Lasten wurden in Kartonboxen mit den Maßen $30 \times 20 \times 20 \mathrm{~cm}$ verpackt. Der Lastschwerpunkt der gefüllten Boxen ist als stabil anzusehen. Um ausreichend Platz zur Entnahme der SKU je Regalebene zu gewährleisten, wurden Fachbodenhöhen auf zwei Regale aufgeteilt und die Regale je nach untersuchtem Fall ausgetauscht.

Zusätzlich wurde im Labor ein Startpunkt markiert, an dem ein Kommissionierwagen bei Versuchsstart platziert ist. Der Kommissionierwagen hat eine Höhe von $90 \mathrm{~cm}$, was nach der VDI-Richtlinie 3657 der optimalen Ablagehöhe entspricht. Der Versuchsaufbau ist zusätzlich in Abb. 3 dargestellt.

Der Versuch wurde mit einer seitlich positionierten Kamera gefilmt. Zur Unterstützung der Erfassung der Rumpfbeugung bzw. -verdrehung wurden die Kommissionieren-

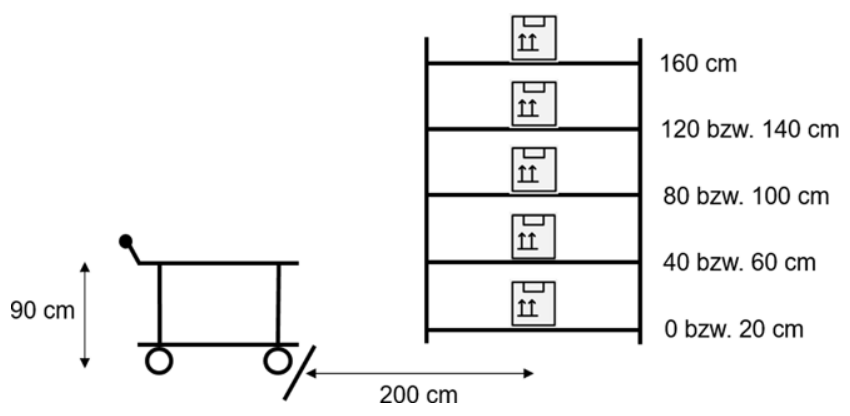

Abb. 3 Versuchsaufbau zur Identifizierung der Körperhaltung bei Kommissioniertätigkeiten

Fig. 3 Experimental set-up for identifying posture during order-picking activities den zudem mit dem IMU-Motion-Capture System TEA Captiv L7000 Premier aufgerüstet.

Versuchsteilnehmende und Versuchsablauf An den Versuchen nahmen insgesamt elf Versuchspersonen teil. Da aus anderen Untersuchungen bekannt ist, dass die Körperhaltung stark von Körpermaßen abhängig ist (Steinebach et al. 2021), wurden Probanden mit deutlich abweichenden Körpergrößen gewählt (Körpergröße $=175,9 \pm 9,7 \mathrm{~cm}$; $\min =160 \mathrm{~cm} ; \max =192 \mathrm{~cm})$. Zudem hat nach Shojaei et al. 2016 auch das Alter einen Einfluss auf das Bewegungsmuster und damit die Körperhaltung bei der Lastaufnahme, sodass ebenso auf eine breite Streuung des Alters unter der Versuchsteilnehmenden geachtet wurde (Alter $=30,2 \pm 11,2$ Jahre; $\min =18$ Jahre; $\max =59$ Jahre). Sieben Versuchspersonen waren männlich und vier weiblich - zwei Personen hatten bereits vorab signifikante Kommissioniererfahrung.

Nachdem die Versuchspersonen bezüglich der Aufgabe instruiert und mit der Messtechnik aufgerüstet wurden, starteten sie mit einem etwa 10-minütigen Übungsdurchlauf, um mit dem Vorgehen vertraut zu werden.

Die Kommissionieraufgabe wurde folgendermaßen definiert: Die Versuchsteilnehmenden schieben den Kommissionierwagen von der markierten Stelle bis zur Entnahmestelle des Regals, entnehmen den entsprechenden Artikel und platzieren ihn auf dem Wagen. Der Umsetzvorgang findet hierbei stets beidhändig statt. Nach Absetzen des Artikels wird der Wagen noch etwa einen Meter weitergeschoben. Dieser Vorgang wird mit jeder Kombination der beiden Einflussgrößen „Greifhöhe“ und „Lastgewicht“ wiederholt, sodass ein vollfaktorieller Versuchsplan mit insgesamt 27 verschiedenen Kommissioniervorgängen vorliegt. Die Reihenfolge der Durchführung wird zwischen den einzelnen Versuchsteilnehmenden gezielt variiert um Reihenfolgeneffekte auszuschließen. Weitere in der Kommissionierung vorkommende (Neben-)Tätigkeiten, wie z.B. die Vereinzelung von Artikeln aus einer Kartonage oder das Scannen von Barcodes, wurden im Versuchsaufbau nicht berücksichtigt. Der konkrete Kommissionierprozess ist hochindividuell und von Unternehmen zu Unternehmen unterschiedlich, sodass sich lediglich auf den Umsetzvorgang konzentriert wurde.

\subsection{Bestimmung der Kompressionskraft mit 3DSSPP}

Ausgangspunkt der Simulation mit 3DSSPP zur Bestimmung der Druckkraft auf die Wirbelsäulensegmente L4-L5 bzw. L5-S1 ist das Videomaterial der Versuchspersonen, die jeweils ein SKU von einer bestimmten Regalebene auf den Handwagen umsetzen. Die drei im Versuch verwendeten Lastgewichte $(0,5 \mathrm{~kg} ; 5 \mathrm{~kg} ; 10 \mathrm{~kg})$ werden jeweils approxi- 


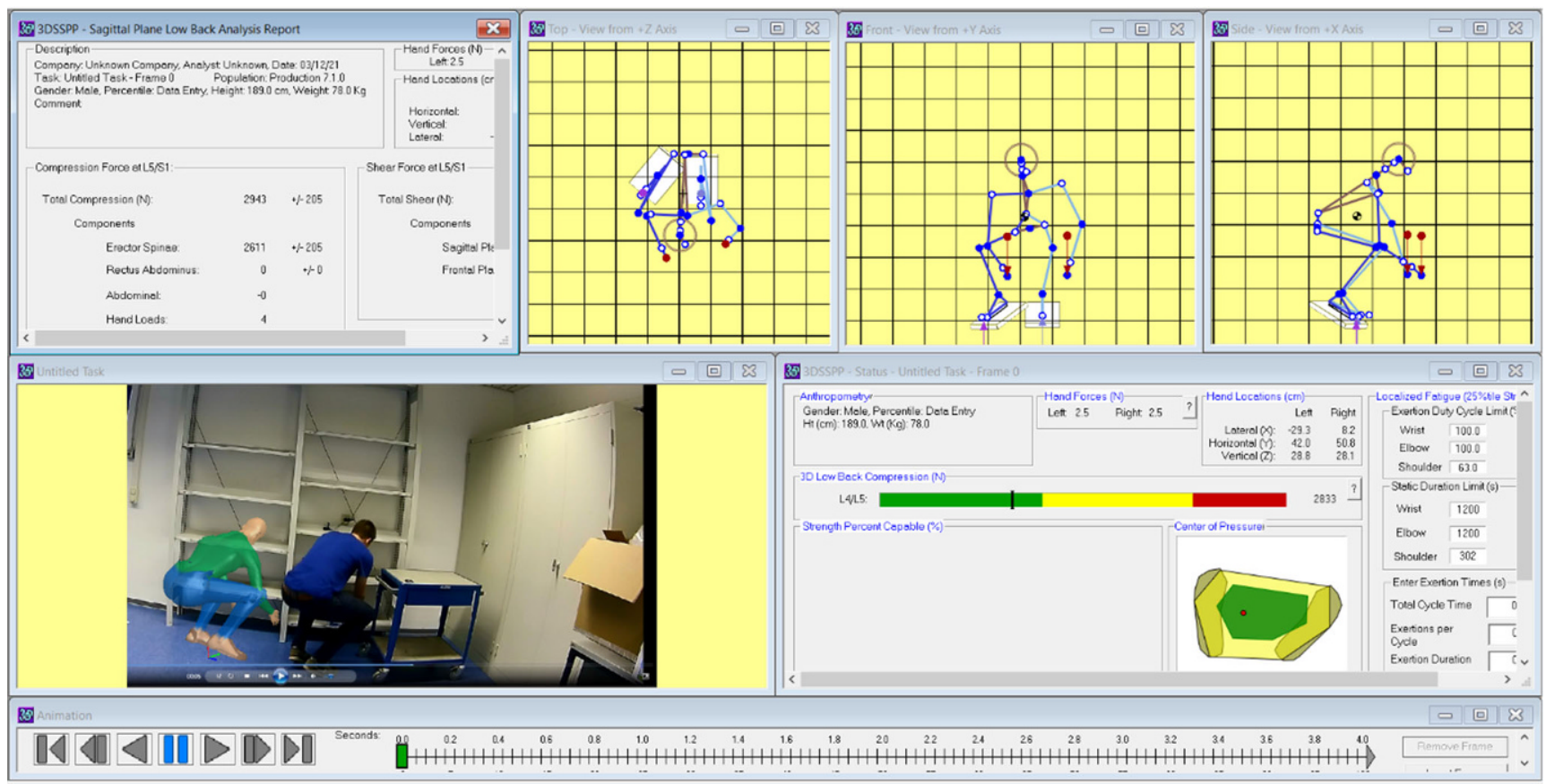

Abb. 4 Ausschnitt aus 3DSSPP - Simulation eines Umsetzvorgangs

Fig. 4 Section of 3DSSPP-simulation of a conversion process

mativ als senkrecht nach unten wirkenden Kraft mit einem Betrag von 5, 50 und $100 \mathrm{~N}$ implementiert, wobei die Gesamtkraft gleichmäßig auf beide Hände verteilt wird.

Die Körperhaltung wird durch die räumliche Lage einzelner Körperteile sowie die sich dadurch ergebenden $\mathrm{Ge}-$ lenkwinkel determiniert (siehe Abb. 4). Entscheidend sind dabei die Ausrichtung von Unter- und Oberschenkel, die Rumpfneigung, die Rumpftorsion, die Schulter-Arm-Stellungen sowie der Lastabstand. Anschließend werden die Gelenkwinkel des Modellavatars unter Zuhilfenahme der Motion Capture Daten iterativ angepasst, bis diese mit den Körperhaltungen der Versuchsteilnehmenden übereinstimmen. Des Weiteren können anthropometrischen Daten der entsprechenden Personen im Programm für die Druckkraftberechnung hinterlegt werden.

Zudem wurde überprüft, zu welchem Zeitpunkt im Umsetzvorgang die maximale Druckkraft auf die beiden untersuchten Wirbelsäulensegmente auftrat, indem die Modellierung in 3DSSPP für verschiedene Zeitpunkte im Bewegungsablauf durchgeführt wurde. Die maximale Kompressionskraft ergab sich in fast jedem Fall zum Zeitpunkt der Lastaufnahme und kann schließlich wie üblich für die gesamte Dauer der Lasthandhabung angenommen werden (vgl. Jäger et al. 2002).

Anschließend wurden die Kenngrößen zur Lumbalbelastung auch für alle anderen Lastgewichte bis $15 \mathrm{~kg}$ in $0,5 \mathrm{~kg}$ Schritten durch Interpolation bestimmt, um diese als Eingangsgrößen für die ergonomische Lagerplatzvergabe nutzen zu können.

\subsection{Ergebnisse und Diskussion der Kommissionierversuche}

In den Abb. 5 bis 7 sind Box-Plot-Diagramme für die mit 3DSSPP ermittelten statischen Druckkräfte auf L5-S1 (in Newton) bei Umsetzvorgängen von drei Lastgewichten aus verschiedenen Regalfachhöhen dargestellt.

Wie zu erwarten war, beeinflusst das zu handhabende Lastgewicht die Druckkraftwerte auf L5-S1 maßgeblich. Beispielsweise steigt die Kompressionskraft bei Umsetzvorgängen von Regalfächern auf $20 \mathrm{~cm}$ Höhe von etwa $2,5 \mathrm{kN}(0,5 \mathrm{~kg})$ auf fast $4 \mathrm{kN}(10 \mathrm{~kg})$. Des Weiteren zeigt sich, dass besonders hohe Druckkraftwerte bei niedrigen Regalfachebenen auftreten. Insbesondere bei Umsetzvorgängen aus niedrigen Regalfachhöhen ist tendenziell mit erhöhter Lumbalbelastung zu rechnen. In den dargestellten Versuchen lagen diese teilweise über den in Abschn. 2.2 erwähnten, empfohlenen maximalen Kompressionskräften. Ursächlich hierfür waren stärkere Rumpfbeugungen der Versuchsteilnehmenden bei den hier vorgegebenen Umsetzvorgängen, um an tief gelagerte SKU zu gelangen. In der Praxis kann es jedoch bei abweichenden Bewegungsmustern oder Prozessen (z.B. Vereinzelung von Artikeln) zu anderen Ergebnissen kommen.

Mit steigender Regalfachhöhe nimmt die Kompressionskraft jedoch tendenziell ab, bis sie ab einer Regalfachhöhe von etwa $100-120 \mathrm{~cm}$ ihr Minimum erreicht. Dies stimmt gut mit der in der VDI 3657 angegebenen optimalen Greifhöhe von $110 \mathrm{~cm}$ überein. Noch höher gelegene Lagerfä- 


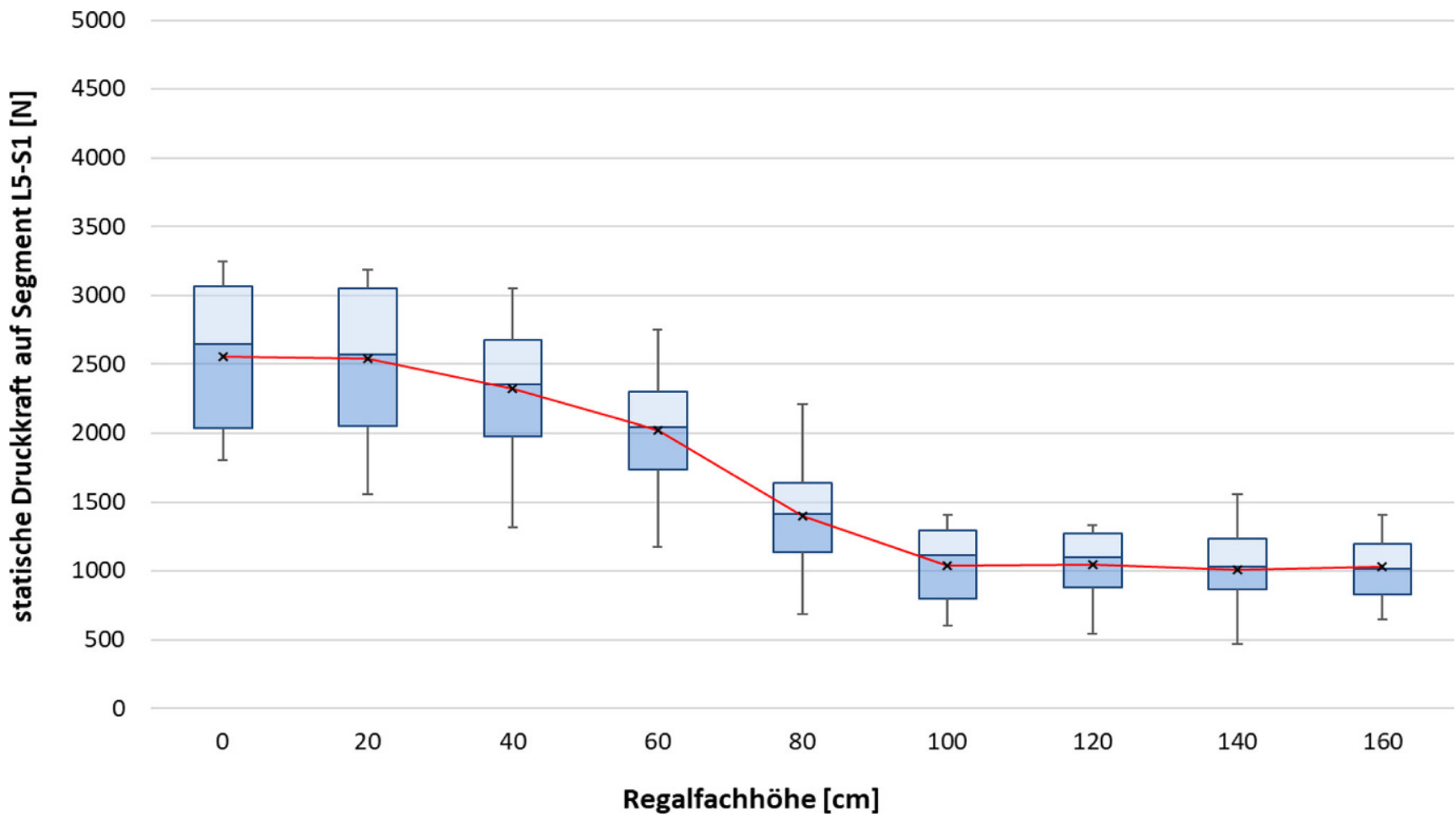

Abb. 5 Statische Druckkraft auf L5-S1 nach 3D SSPP bei Kommissioniervorgängen aus verschiedenen Regalfachhöhen bei Lastgewichten mit $0,5 \mathrm{~kg}$

Fig. 5 Static compressive force on L5-S1 according to 3D SSPP during picking operations from different shelf heights at load weights with $0.5 \mathrm{~kg}$

cher unterscheiden sich bezüglich der Kompressionskraft nicht mehr signifikant von den Werten auf 100 bzw. $120 \mathrm{~cm}$ und bleiben in etwa auf einem konstanten Niveau. Hierdurch wird deutlich, dass für die Ermittlung der Druckkraft in 3DSSPP neben dem Lastgewicht vor allem der sagittale Beugungswinkel des Rumpfes entscheidend ist, da es ab einer Regalfachhöhe von etwa $100 \mathrm{~cm}$ kaum noch zu Rumpfbeugung kommt.

Wie bereits in Steinebach et al. (2021) gezeigt werden konnte, hat das Lastgewicht hingegen einen eher geringen Einfluss auf die Körperhaltung. Obwohl der Verlauf der Druckkraftmittelwerte für alle drei Lastgewichte deshalb recht ähnlich ist, fällt auf, dass die Differenz zwischen den höchsten und kleinsten Druckkraftmittelwerten innerhalb einer Lastgewichtsklasse mit steigendem Lastgewicht zunimmt. So wird deutlich, dass ein ergonomisch günstiger Lagerplatz insbesondere für sehr schwere SKU wichtig ist, um Überbelastungen vermeiden zu können.

Ferner sind die starken Schwankungen der Kompressionskraft auf L5-S1 in tiefen Regalebenen auffällig, was durch unterschiedliche Bewegungsmuster erklärt werden kann. Während manche Versuchspersonen die Artikel eher „aus dem Rücken“ heben, gehen andere stärker in die Knie (siehe Abb. 4). Bei den etwas schwereren Lastgewicht tendieren die Versuchspersonen eher dazu, beim Heben in die Knie zu gehen, sodass die Schwankungen etwas niedriger sind und auch die mittlere Druckkraft etwas niedriger ausfällt, als dies bei einem „Heben aus dem Rücken“ zu erwarten wäre.

Allgemein ist anzumerken, dass die Werte bezüglich der Wirbelsäulensektion L4-L5 sehr ähnliche Verläufe haben, weshalb auf eine detaillierte Darstellung verzichtet wird.

Im Vergleich mit der in Abschn. 2.2 erwähnten Literatur ergeben sich bei Goldscheid (2008) beim Kommissionieren höhere Druckkraftwerte im Bereich der hohen Entnahmebereiche im Vergleich mit jenen in mittleren Höhen. Die Größenordnung der hier berechneten Mittelwerte für die unteren Ebenen liegen jedoch in einem sehr ähnlichen Bereich. Ebenso lassen sich die Werte mit jenen von Jäger et al. (2002) vergleichen. Bei einem standardisierten Hebevorgang von 10 auf $90 \mathrm{~cm}$ ergeben sich in Abhängigkeit vom Zeitverlauf des Hebens (Berücksichtigung der Massenträgheit) bei $10 \mathrm{~kg}$ interpolierte Werte zwischen $2,41 \mathrm{kN}$ (statisch) und 4,01 kN (dynamisch; 1,0 s Ausführungszeit). Die Kompressionskräfte aus Jäger et al. (2002) sind entsprechend für das statische Heben im Vergleich mit den in diesem Versuch ermittelten Werten (etwa $3,8 \mathrm{kN}$ beim Heben von 10 auf $90 \mathrm{~cm}$ ) niedriger einzuschätzen, da die Versuchsteilnehmer im vorliegenden Beitrag teilweise stärkere Rumpfbeugung oder unsymmetrische Körperhaltungen eingenommen haben. Ebenso lässt sich die Bedeutung des hier vernachlässigten Effekts der Massenträgheit erkennen. 


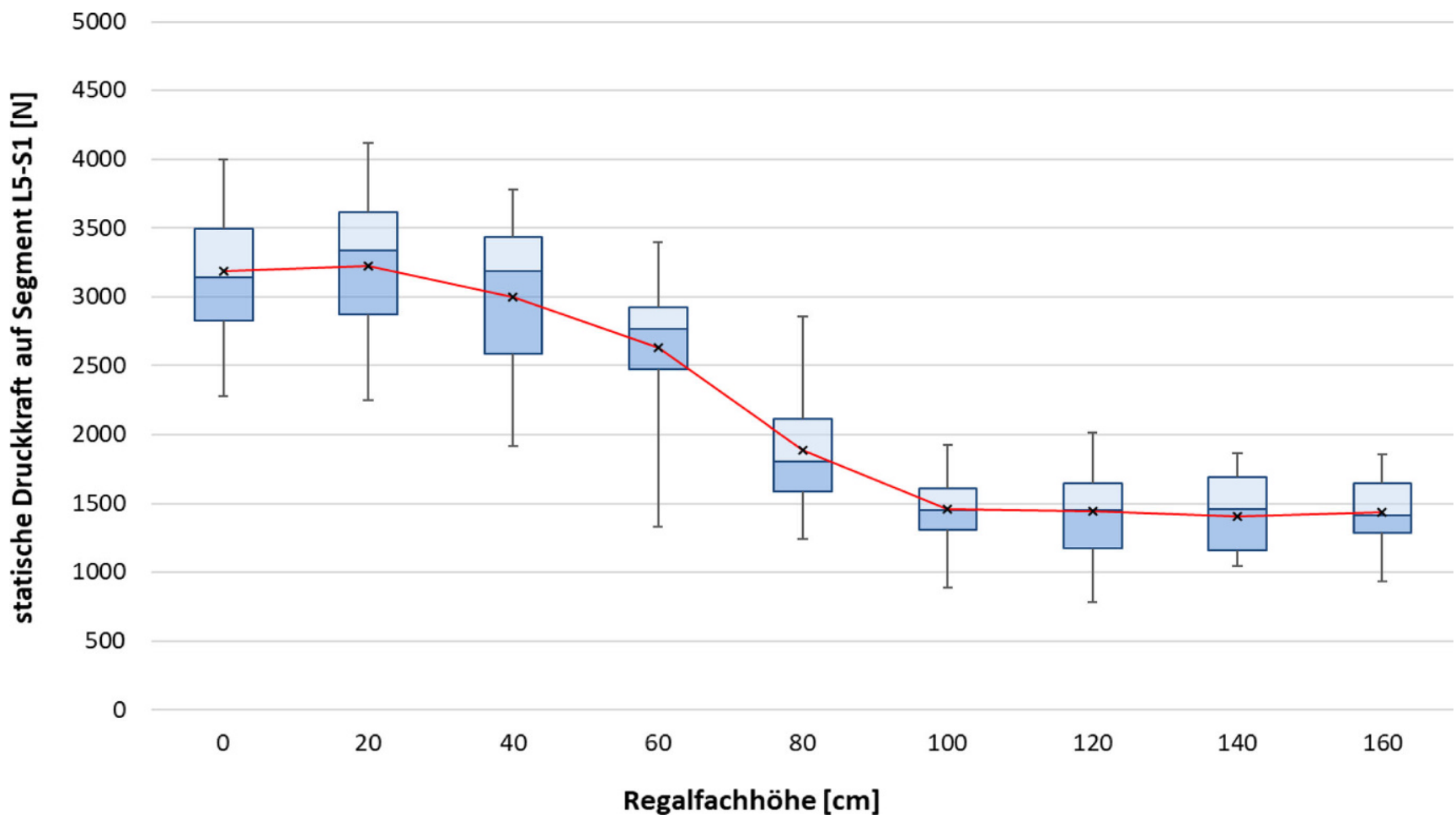

Abb. 6 Statische Druckkraft auf L5-S1 nach 3D SSPP bei Kommissioniervorgängen aus verschiedenen Regalfachhöhen bei Lastgewichten mit $5 \mathrm{~kg}$

Fig. 6 Static compressive force on L5-S1 according to 3D SSPP during picking operations from different shelf heights with load weights of $5 \mathrm{~kg}$

$\mathrm{Zu}$ beachten sind auch einige Limitationen der hier ermittelten Kenngrößen. Zum einen sind in 3DSSPP keine dynamischen Effekte, wie beispielsweise die Wirkung der Massenträgheit, in den Druckkräften berücksichtigt. Zu erwarten ist, dass die wahren Werte entsprechend höher sind. Aufgrund der großen Schwankungen und der verschiedenen Bewegungsmuster, sind die hier dargestellten Resultate als Tendenzen zu verstehen, um die Wirksamkeit einer potenziell auf diesen Kennwerten basierenden Lagerplatzvergabe $\mathrm{zu}$ verstehen. Eine rückwirkende Exakte Bestimmung der Lumbalbelastung bei gegebener LPV soll mit dem folgenden Algorithmus nicht ermöglicht werden. Zudem ist die Druckkraft auf L5-S1 (bzw. Scherkraft) nicht der einzige Kennwert, der für die physische Belastung bei Kommissioniervorgängen relevant ist. Dies wird vor allem in den höheren Lagerfächern deutlich, die entsprechend der Kompressionskräfte nicht kritischer bewertet werden, als Lagerplätze in der „Goldenen Zone“, obwohl die Versuchspersonen hier Arbeit auf oder über Schulterhöhe durchführen. Energetische, muskuläre oder auch psychophysiologische Kriterien könnten hier ebenfalls eine Rolle spielen.

\section{Herleitung einer bikriteriellen LPV- Optimierung}

Im Folgenden soll auf Basis der zuvor festgelegten Kenngrößen eine bikriterielle Optimierung der Lagerplatzzuordnung für manuelle Kommissioniersysteme mit Fachbodenregalen hergeleitet werden. Hierbei wird davon ausgegangen, dass die Kommissionierenden an einem Depot starten und nach dem Einsammeln der SKU zum Ausgangsort zurückkehren. Der Einfachheit halber wird angenommen, dass der Artikel nah an der vorderen Kante des Regals platziert ist und ein tiefes Hineingreifen (analog zu den Kommissionierversuchen) entfällt. Die Bewegung durch das Lager kann entweder mit einem manuellen Handwagen oder einem Fahrzeug stattfinden (z.B. Niederhubkommissionierer). Zudem soll der Algorithmus auf jegliche Lagerlayouts und Regaltypen anwendbar sein.

\subsection{Definition der Kennzahlen und Variablen}

Das Ziel der LPV-Optimierung ist die Zuordnung einer Menge I von SKU zu einer Menge J von Lagerplätzen, wobei hinsichtlich zweier Zielfunktionen - die Minimierung der lumbalen Belastung über die Belastungskennwerte $l b_{i, j}$ sowie die Minimierung der Kommissionierzeit $t_{i, j}-$ mit- 


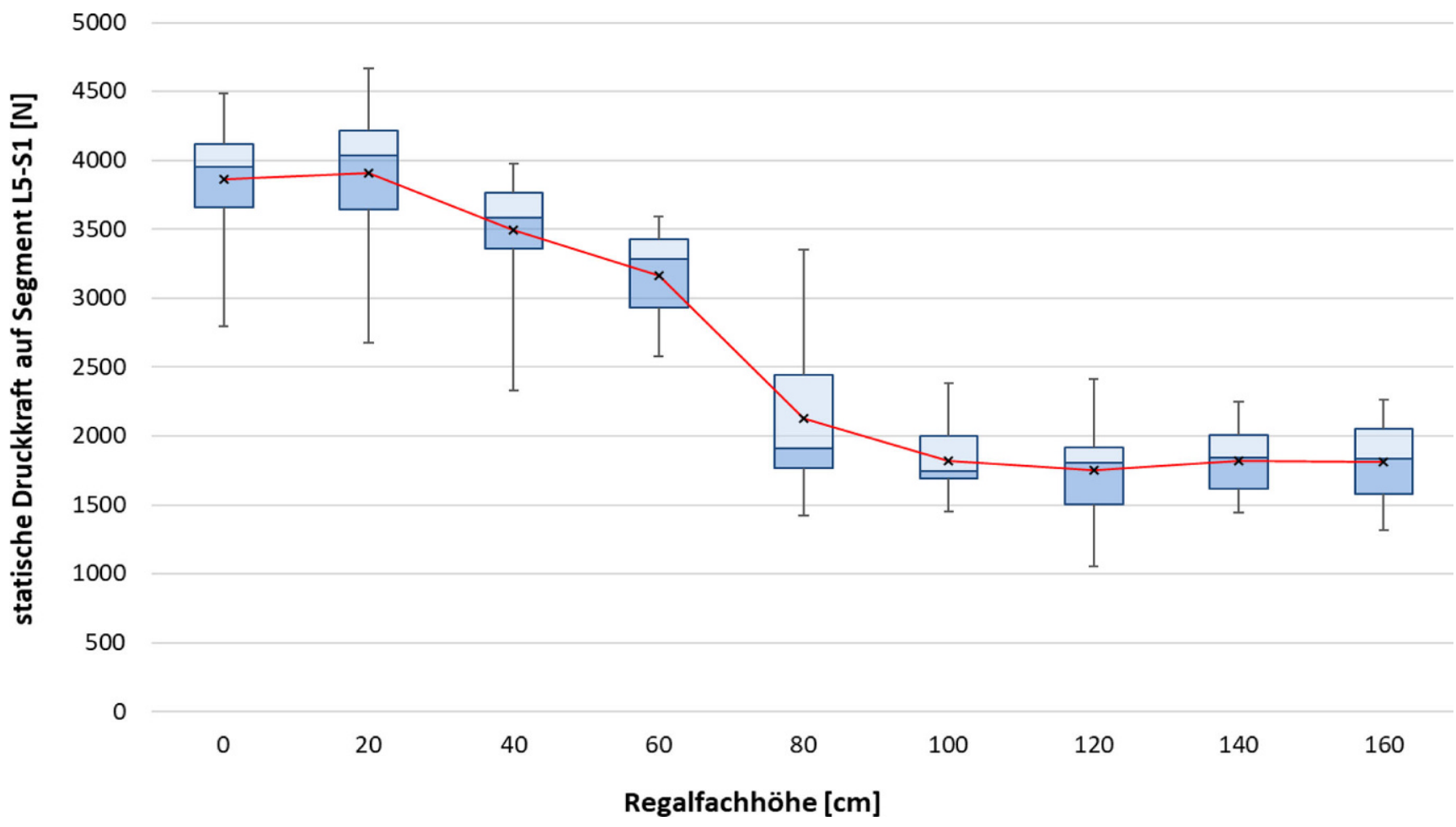

Abb. 7 Statische Druckkraft auf L5-S1 nach 3D SSPP bei Kommissioniervorgängen aus verschiedenen Regalfachhöhen bei Lastgewichten mit $10 \mathrm{~kg}$

Fig. 7 Static compressive force on L5-S1 according to 3D SSPP during picking operations from different shelf heights with load weights of $10 \mathrm{~kg}$

tels eines Algorithmus pareto-optimale Lösungen gefunden werden sollen.

Hierbei beschreibt die Variable $l b_{i, j}$ die Lumbalbelastung, ausgedrückt über die Kompressionskraftdosis auf das Wirbelsäulensegment L5-S1, falls SKU i aus Lagerplatz j auf den Kommissionierwagen umgesetzt wird. Die Druckkraftdosis ergibt sich aus den Kennzahlen der Kommissionierversuche für je eine Kombination aus Lastgewicht und Regalfachhöhe, welche entsprechend dem Mainz-Dortmunder Dosismodell (Hartung et al. 1999; Jäger et al. 1999) zu einer Druckkraftdosis verrechnet werden. Analog dazu ist $t_{i, j}$ definiert als die benötigte Kommissionierzeit (Wegzeit und Greifzeit) von SKU i aus Lagerplatz j. Die entsprechenden Kennzahlen $t_{i, j}$ werden mittels MTM-UAS ermittelt.

Zudem werden für die Optimierung eine Reihe weiterer Variablen genutzt, welche in Tab. 1 zusammengefasst sind.

\subsection{Formulierung des Lagerplatzvergabe- Algorithmus}

Zunächst werden die Zielfunktionen der Optimierung für beide Zielgrößen getrennt voneinander formuliert, um hiermit je eine Zuordnung mit minimaler Lumbalbelastung
$L B^{0}$ und minimaler Kommissionierzeit $T^{0}$ berechnen zu können:

Minimiere $L B=\sum_{i=1}^{I} \sum_{j=1}^{J} l b_{i j} \cdot x_{i j} \cdot n_{i}$

Minimiere $T=\sum_{i=1}^{I} \sum_{j=1}^{J} t_{i j} \cdot x_{i j} \cdot n_{i}$

mit $x_{i, j}=\left\{\begin{array}{c}1, \text { wennSKUiauf Lagerplatz j gelagertwird } \\ 0 \text { sonst }\end{array}\right.$

Hierbei entscheidet die Binärvariable $x_{i j}$ darüber, ob ein bestimmtes SKU i einem definierten Lagerplatz $\mathrm{j}$ zugeordnet wird, sodass in den jeweiligen Zielfunktionen nur die Druckkraftdosen bzw. Zeitanteile aufsummiert werden, die entsprechend der LPV tatsächlich vorkommen. Da ein SKU in einer bestimmten Zeitspanne auch mehrfach kommissioniert werden kann, müssen die Kennzahlen mit der Zugriffshäufigkeit $n_{i}$ multipliziert werden. 
Tab. 1 Variablen und Parameter zur bikriteriellen LPV-Optimierung Table 1 Variables and parameters for bicriteria optimisation of bin management

\begin{tabular}{ll}
\hline Index & Bedeutung \\
\hline $\mathrm{i}$ & SKU-Index mit $\mathrm{i}=1, \ldots, \mathrm{I}$ \\
$\mathrm{j}$ & Lagerplatz-Index mit $\mathrm{j}=1, \ldots, \mathrm{J}$ \\
$\mathrm{I}$ & Gesamtanzahl an SKU \\
$\mathrm{J}$ & Gesamtanzahl an Lagerplätzen \\
$n_{i}$ & Zugriffshäufigkeit des SKU i je Zeitperiode \\
$x_{i j}$ & Binäre Entscheidungsvariable zur Lagerung von \\
& SKU i auf Lagerplatz j \\
$t_{\text {Weg, } i j}$ & Wegzeit, um zu SKU i an Lagerplatz j zu gelangen \\
$t_{\text {Greif }, i j}$ & Greifzeit, um SKU i aus Lagerplatz j zu entnehmen \\
$t_{i j}$ & Gesamte Kommissionierzeit von SKU i auf Lager- \\
$l b_{i j}$ & platz j \\
$L B^{0}$ & Kennwert zur Druckkraftdosis auf L5-S1 von SKU i \\
$T^{0}$ & auf Lagerplatz j \\
$\lambda$ & Minimale lumbale Gesamtbelastung \\
\hline$L B$ & Minimale Gesamtkommissionierzeit \\
$\bar{T}$ & Gewichtungsfaktor $\in[0,1]$ \\
\hline
\end{tabular}

Zudem sind in dem Optimierungsmodell Nebenbedingungen einzuhalten:

$$
\begin{array}{ll}
\sum_{j=1}^{J} x_{i j}=1 & \forall i \\
\sum_{i=1}^{I} x_{i j}=1 & \forall j \\
x_{i, j} \in\{0 ; 1\} &
\end{array}
$$

Die oberen beiden Nebenbedingungen stellen sicher, dass jedem Lagerplatz j nur genau ein SKU i zugeordnet wird und umgekehrt, um Mehrfachzuordnungen $\mathrm{zu}$ vermeiden.

Die somit aufgestellte bikriterielle Optimierung aus den Zielgrößen „lumbale Belastung“ und „Kommissionierzeit“ sollen nun in ein einkriterielles Modell überführt werden. Da zu vermuten ist, dass die beiden Größen in einem Zielkonflikt stehen, gilt es pareto-optimale Lösungen zu finden. Der am weitesten verbreitete Ansatz, um multikriterielle Zielfunktionen in einkriterielle Ansätze umzuformen ist die Gewichtete-Summen-Methode (Marler und Arora 2004). Voraussetzung für deren Anwendung ist die Normierung der Zielfunktionen zu dem Zweck, diese dimensionslos und damit vergleichbar zu machen. Diese dimensionslosen Größen können erzeugt werden, indem man die oben genannten Zielfunktionen in Relation zum errechneten Optimalwert der jeweiligen Zielgröße setzt:

$\overline{L B}=\frac{\left(\sum_{i=1}^{I} \sum_{j=1}^{J} l b_{i j} \cdot x_{i j} \cdot n_{i}\right)-L B^{0}}{L B^{0}}$
$\bar{T}=\frac{\left(\sum_{i=1}^{I} \sum_{j=1}^{J} t_{i j} \cdot x_{i j} \cdot n_{i}\right)-T^{0}}{T^{0}}$

Die sich hieraus ergebende neue Zielfunktion lautet:

Minimiere $F=\lambda \cdot \overline{L B}+(1-\lambda) \cdot \bar{T}$

Es gelten die gleichen Nebenbedingungen wie in dem zuvor aufgestellten bikriteriellen Optimierungsproblem. Mittels einem von den Anwendenden bzw. Lagerplanenden festzulegenden Gewichtungsfaktor $\lambda(0 \leq \lambda \leq 1)$ kann nun eine LPV durch Minimierung der Funktion F gefunden werden, die beide ursprünglichen Zielfunktionen pareto-optimal löst. Hierbei nimmt die kumulative Kompressionskraft auf L5-S1 mit steigendem $\lambda$ ab. In einem so gefundenen Pareto-Optimum ist (bei konstantem $\lambda$ ) eine weitere Reduzierung der Druckkraftdosis nur noch durch eine Erhöhung der Kommissionierzeit möglich und umgekehrt. Da diese pareto-optimale Lösung jedoch für jeden Gewichtungsfaktor $\lambda$ unterschiedlich ist, gibt es eine Menge an „Kompromisslösungen“, welche sich in einer Pareto-Kurve abbilden lassen (siehe Abschn. 5.3).

Das Optimierungsmodell wurde mit der Programmiersprache Java in der Entwicklungsumgebung Eclipse erstellt und unter Zuhilfenahme des Softwarepakets ILOG CPLEX Optimization Studie von IBM mit einem Branch-and-Cut Verfahren gelöst.

\subsection{Anwendung der ergonomischen LPV auf einen beispielhaften Datensatz}

Durch Anpassung der Kennzahlen $l b_{i j}$ und $t_{i j}$ kann der Algorithmus auf beliebige Lagerlayouts und Regaltypen angepasst werden. Beispielhaft soll die ergonomische LPV auf ein one-Block Layout (Abb. 8) mit acht Regalreihen angewandt werden. Die Regale besitzen in diesem Beispiel fünf Fachböden auf den Höhen $0 \mathrm{~cm}, 40 \mathrm{~cm}, 80 \mathrm{~cm}, 120 \mathrm{~cm}$ und $160 \mathrm{~cm}$. Insgesamt finden 520 verschiedene Artikel in diesem Lager Platz. Die Artikeleigenschaften sind hierbei gleichverteilt mit Lastgewichten zwischen 0,5 und $10 \mathrm{~kg}$ sowie Zugriffshäufigkeiten zwischen 1 und 10 pro Tag.

Das SKU-Portfolio mit 520 Artikeln wurde mittels der entwickelten ergonomischen LPV für verschiedene $\lambda$ auf die Lagerplätze des beschriebenen Layouts zugeordnet. Abb. 9 zeigt die entsprechende Pareto-Kurve mit zwei beispielhaften Steigungsdreiecken.

Der Pareto-Kurve ist zu entnehmen, dass sowohl bezüglich der Kommissionierzeit, als auch bezüglich der Druck- 


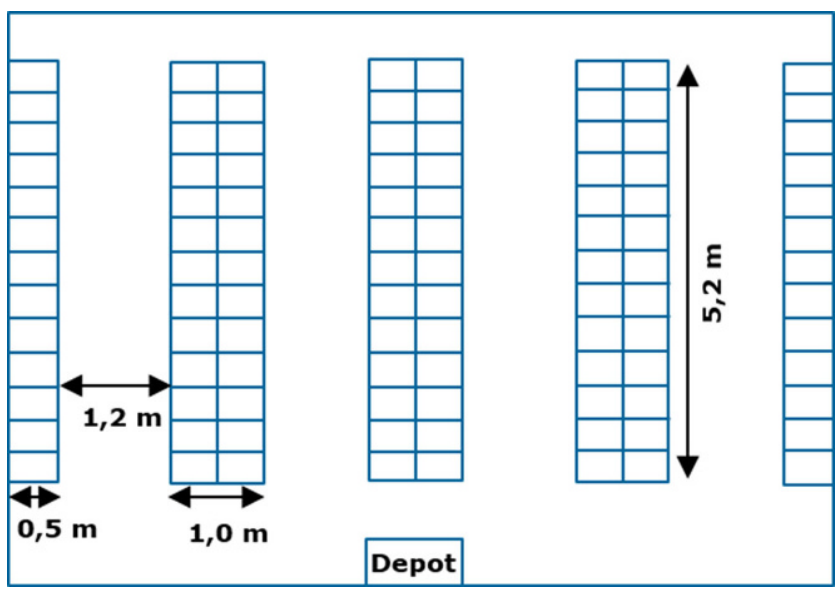

Abb. 8 Lagerlayout bezüglich der beispielhaften ergonomischen LPV Fig. 8 Warehouse layout with regard to exemplary ergonomic storage location management

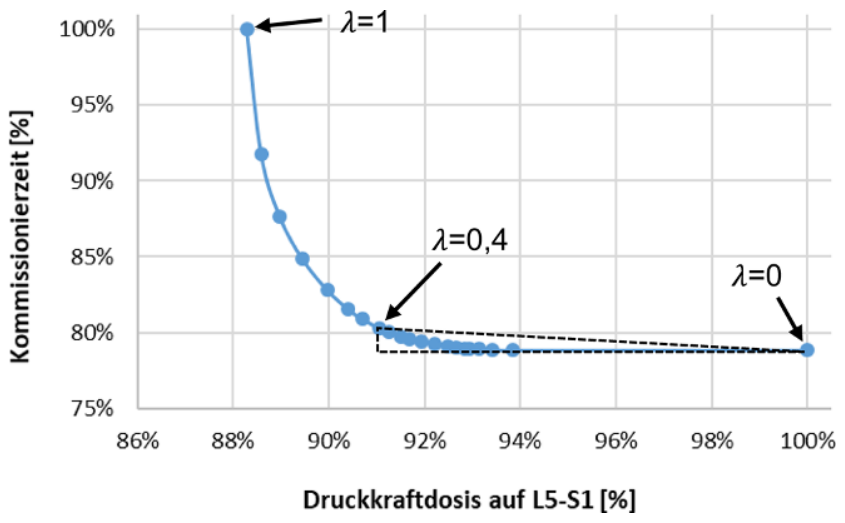

Abb. 9 Pareto-Kurve des Beispieldatensatzes bei Variation von $\lambda$ Fig. 9 Pareto curve of the example data set with variation of $\lambda$

kraftdosis auf L5-S1 Reduzierungspotentiale vorliegen. Bei einer Einzeloptimierung der beiden Zielgrößen $(\lambda=0$ bzw. $\lambda=1)$ können durch eine Variation der LPV etwa $21 \%$ an Kommissionierzeit eingespart bzw. die Druckkraftdosis um etwa $12 \%$ reduziert werden. Diese Ergebnisse stimmen gut mit der eingangs verwendeten Literatur überein, wobei die Reduzierungspotentiale bei Battini et al. 2016 mit max. $7 \%$ (Energieumsatz) bzw. $7 \%$ (Zeit) niedriger und bei Larco et al. 2017 mit 19\% (Diskomfort) bzw. $27 \%$ (Zeit) etwas höher liegen.

Zudem wird klar, dass die beiden Größen im Zielkonflikt stehen. Bei einer zeitminimalen Zuordnung werden die häufig zu pickenden SKU nahe des Depots angeordnet, sodass ggf. auch sehr schwere Artikel auf tief gelegenen Regalebenen eingelagert werden. Anders herum werden bei einer belastungsminimalen Zuordnung schwere und häufig gegriffene Artikel auf günstige Regalebenen verteilt, sodass längere Gehwege entstehen. Auffällig ist, dass bei einer Minimierung der einen Zielgröße die jeweils andere sehr hohe
Werte aufweist. Mit der bikriteriellen Optimierung lassen sich hingegen attraktive Kompromisslösungen finden, was durch das rechte Steigungsdreieck in Abb. 8 verdeutlicht werden soll. Ausgehend von einer zeitoptimalen Lösung $(\lambda=0)$ kann durch eine Erhöhung auf $\lambda=0,4$ eine relativ gute Reduzierung der Lumbalbelastung bei gleichzeitig sehr geringer Erhöhung der Kommissionierzeit erreicht werden. Entsprechend kann abgeleitet werden, dass eine Optimierung nach nur einer der beiden Zielgrößen nicht zu empfehlen ist.

\section{Fazit und Ausblick}

In diesem Beitrag konnte eine ergonomische Lagerplatzvergabe hergeleitet werden, welche entsprechend zweier Zielgrößen bikriteriell optimiert wird. Hierzu wurden zunächst Kenngrößen für die benötigte Kommissionierzeit entsprechend MTM-UAS hergeleitet. Da Schädigungen des unteren Rückens bei Kommissionierenden besonders häufig vorkommen, sollte als Belastungskenngröße die Druckkraft auf das Wirbelsäulensegment L5-S1 dienen. $\mathrm{Zu}$ diesem Zweck wurden in einem Labor Kommissionierversuche durchgeführt und die von Versuchspersonen eingenommenen Körperhaltungen in dem biomechanischen Simulationsprogramm 3DSSPP implementiert. Mit einem Gewichtete-Summen-Ansatz wurden beide Probleminstanzen zu einer einkriteriellen Optimierung zusammengefügt und pareto-effizient gelöst, wobei aufgrund des Zielkonflikts gerade Kompromisslösungen aus beiden Zielgrößen besonders attraktiv erscheinen.

Die hier dargestellten Resultate, insbesondere die Bestimmung der Kennwerte zur Lumbalbelastung bei Kommissioniertätigkeiten, sind aufgrund der geringen Probandenzahl, des wenig komplexen Tools 3DSSPP und der groBen Streuung der Werte zunächst nur als tendenzielle Ergebnisse zu betrachten, um die methodische Herleitung eines LPV-Algorithmus aufzuzeigen. Zudem wurden ,standardisierte“ Artikelgrößen verwendet, die im Versuch nur beidhändig gegriffen werden durften - in der Praxis ist dies sicherlich meist nicht gegeben. In weiteren Untersuchungen sollten diese weiter evaluiert werden und beispielsweise auch dynamische Effekte, Scherkraftkomponenten oder energetische Belastungskriterien Berücksichtigung finden. Hierfür bieten sich auch Screening-Verfahren, wie z.B. das EAWS-Verfahren an, um ganzheitlich ergonomische Risiken nach erfolgter Lagerplatzvergabe zu bewerten. Des Weiteren ist es sinnvoll, die entwickelte LPV in einer realen Anwendungsumgebung oder einem Laborversuch mit erfahrenen Arbeitspersonen zu evaluieren.

Funding Open Access funding enabled and organized by Projekt DEAL. 
Open Access Dieser Artikel wird unter der Creative Commons Namensnennung 4.0 International Lizenz veröffentlicht, welche die Nutzung, Vervielfältigung, Bearbeitung, Verbreitung und Wiedergabe in jeglichem Medium und Format erlaubt, sofern Sie den/die ursprünglichen Autor(en) und die Quelle ordnungsgemäß nennen, einen Link zur Creative Commons Lizenz beifügen und angeben, ob Änderungen vorgenommen wurden.

Die in diesem Artikel enthaltenen Bilder und sonstiges Drittmaterial unterliegen ebenfalls der genannten Creative Commons Lizenz, sofern sich aus der Abbildungslegende nichts anderes ergibt. Sofern das betreffende Material nicht unter der genannten Creative Commons Lizenz steht und die betreffende Handlung nicht nach gesetzlichen Vorschriften erlaubt ist, ist für die oben aufgeführten Weiterverwendungen des Materials die Einwilligung des jeweiligen Rechteinhabers einzuholen.

Weitere Details zur Lizenz entnehmen Sie bitte der Lizenzinformation auf http://creativecommons.org/licenses/by/4.0/deed.de.

\section{Literatur}

Battini D, Glock CH, Grosse EH, Persona A, Sgarbossa F (2016) Human energy expenditure in order picking storage assignment: a biobjective method. Comput Ind Eng 94:147-157

Bernnat R (1997) Strategien der Lagerplatzvergabe: Rationalisierungspotentiale im Kommissionierlager. Springer, Berlin, Hiedelberg, New York

Bokranz R, Landau K (2012) Handbuch Industrial Engineering. Produktivitätsmanagement mit MTM. Schäffer-Poeschel, Stuttgart

Borg G (1982) A category scale with ratio properties for intermodal and interindividual comparisons. Psychophys Judgm Process Percept 25-34

Bullinger-Hoffmann AC, Mühlstedt J (2016) Homo sapiens digitalis Virtuelle Ergonomie und digitale Menschmodelle. Springer Vieweg, Wiesbaden

Calzavara M, Glock CH, Grosse EH, Sgarbossa F (2019) An integrated storage assignment method for manual order picking warehouses considering cost, workload and posture. Int J Prod Res 57(8):2392-2408

De Koster R, Le-Duc T, Roodbergen KJ (2007) Design and control of warehouse order picking: a literature review. Eur J Oper Res 182(2):481-501

DGUV (2020) MEGAPHYS: Mehrstufige Gefährdungsanalyse physischer Belastungen am Arbeitsplatz (DGUV Report 3/2020). Abschlussbericht zum Kooperationsprojekt von BAuA und DGUV, Bd. 2

Diefenbach H, Glock CH (2019) Ergonomic and economic optimization of layout and item assignment of a $\mathrm{U}$-shaped order picking zone. Comput Ind Eng 138:106094

Elbert R, Franzke T, Glock CH, Grosse EH (2015) Agent-based analysis of picker blocking in manual order picking systems: effects of routing combinations on throughput time. In: Winter Simulation Conference (WSC). IEEE, , S 3937-3948

Gajšek B, Dukić G, Butlewski M, Opetuk T, Cajner H, Kač SM (2020) The impact of the applied technology on health and productivity in manual "picker-to-part" systems. Work 65(3):1-12

Galka S, Günthner WA (2016) Einfluss von Artikeleigenschaften auf die Entnahmezeit in Kommissioniersystemen. Logist J Proc

Garg A, Chaffin DB, Herrin GD (1978) Prediction of metabolic rates for manual materials handling jobs. Am Ind Hyg Assoc J 39(8):661-674

Glock CH, Grosse EH, Abedinnia H, Emde S (2019) An integrated model to improve ergonomic and economic performance in order picking by rotating pallets. Eur J Oper Res 273(2):516-534

Goldscheid C (2008) Ermittlung der Wirbelsäulenbelastung in manuellen Kommissioniersystemen. Shaker,
Grosse EH, Glock CH, Neumann WP (2015) Human factors in order picking system design: a content analysis. IFAC-PapersOnLine 48(3):320-325

Grosse EH, Glock CH, Neumann WP (2017) Human factors in order picking: a content analysis of the literature. Int J Prod Res 55(5): $1260-1276$

Gudehus T (2012) Logistik 2: Netzwerke, Systeme und Lieferketten. Springer, Berlin, Heidelberg, New York

Gue KR, Meller RD, Skufca JD (2006) The effects of pick density on order picking areas with narrow aisles. IIE Trans 38(10):859-868

Hanson R, Medbo L, Assaf M, Jukic P (2018) Time efficiency and physical workload in manual picking from large containers. Int J Prod Res 56(3):1109-1117

Hartung E, Schäfer K, Jäger M, Luttmann A, Bolm-Audorff U, Kuhn S, Francks HP (1999) Mainz-Dortmunder Dosismodell (MDD) zur Beurteilung der Belastung der Lendenwirbelsäule durch Heben oder Tragen schwerer Lasten oder durch Tätigkeiten in extremer Rumpfbeugehaltung bei Verdacht auf Berufskrankheit Nr. 2108. Teil 2: Vorschlag zur Beurteilung der arbeitstechnischen Voraussetzungen im Berufskrankheiten-Feststellungsverfahren. Arbeitsmed Sozialmed Umweltmed 34:112-122

ten Hompel M, Sadowsky V, Beck M (2011) Kommissionierung: Materialflusssysteme 2 - Planung und Berechnung der Kommissionierung in der Logistik. Springer, Berlin, Heidelberg, New York

Jäger M, Luttmann A, Bolm-Audorff U, Schäfer K, Hartung E, Kuhn S, Francks HP (1999) Mainz-Dortmunder Dosismodell (MDD) zur Beurteilung der Belastung der Lendenwirbelsäule durch Heben oder Tragen schwerer Lasten oder durch Tätigkeiten in extremer Rumpfbeugehaltung bei Verdacht auf Berufskrankheit Nr. 2108. Teil 1: Retrospektive Belastungsermittlung für risikobehaftete Tätigkeitsfelder. Arbeitsmed Sozialmed Umweltmed 34:101-111

Jäger M, Luttmann A, Göllner R, Laurig W (2000) Der Dortmunder: Biomechanische Modellbildung zur Bestimmung und Beurteilung der Belastung der Lendenwirbelsäule bei Lastenhandhabungen. Prävention von arbeitsbedingten Gesundheitsgefahren und Erkrankungen. Monade, Leipzig, S 105-124

Jäger M, Gollner R, Jordan C, Theilmeier A, Luttmann A (2002) Belastung der Lendenwirbelsäule beim Heben und Umsetzen von Lasten. Z Arb Wiss 56(1/2):93-105

Jäger M, Geiß O, Bergmann A, Bolm-Audorff U, Ditchen D, Ellegast R, Luttmann A (2007) Biomechanische Analysen zur Belastung der Lendenwirbelsäule innerhalb der Deutschen Wirbelsäulenstudie. Zbl Arbeitsmed 57(9):264-276

Jordan C, Jäger M, Theilmeier A, Luttmann A (2001) Wirbelsäulenbelastung bei ausgewählten Tragetätigkeiten. Z Arbeitswiss $55: 144-153$

Karhu O, Kansi P, Kuorinka I (1977) Correcting working postures in industry: a practical method for analysis. Appl Ergon 8(4):199-201

Kretschmer V, Reiser J, Rinkenauer G (2021) Stellenwert der Kognitiven Ergonomie in cyber-physischen Logistiksystemen: Ergebnisse einer Feldstudie bei einem Versandhandelsunternehmen. In: GfA Frühjahreskongress 2021. GfA Press,

Kuhlang P (2014) Zeitwirtschaft in Lean Productionund Lean Logistics. In: StEP-Up Best Practice Konferenz

Larco JA, De Koster R, Roodbergen KJ, Dul J (2017) Managing warehouse efficiency and worker discomfort through enhanced storage assignment decisions. Int J Prod Res 55(21):6407-6422

Marler RT, Arora JS (2004) Survey of multi-objective optimization methods for engineering. Struct Multidiscip Optim 26(6):369-395

Michel R (2017) 2017 Warehouse/DC Operations Survey. In the thick of e-commerce adjustments. Logist Manag 56(11):52-58

Napolitano M (2012) 2012 warehouse/DC operations survey: mixed signals. Mod Mater Handl 51(11):48-56

Otto A, Boysen N, Scholl A, Walter R (2017) Ergonomic workplace design in the fast pick area. OR Spectr 39(4):945-975 
Petersen CG, Siu C, Heiser DR (2005) Improving order picking performance utilizing slotting and golden zone storage. Int J Oper Prod Manag 25(10):997-1012

Qin K, Chen FY, Ma L (2015) Cutting down the travel distance of put systems at Kunming International Flower Auction Market. Int J Prod Res 53(12):3573-3585

Reyes J, Solano-Charris E, Montoya-Torres J (2019) The storage location assignment problem: a literature review. Int J Ind Eng Comput 10(2):199-224

Schlick C, Bruder R, Luczak H (2018) Arbeitswissenschaft. Springer, Berlin, Heidelberg, New York

Shojaei I, Vazirian M, Croft E, Nussbaum MA, Bazrgari B (2016) Age related differences in mechanical demands imposed on the lower back by manual material handling tasks. J Biomech 49(6):896-903

Spath D, Ehmann R, Braun M, Kille B, Marrenbach D, Scholtz O (2019) Der Mensch in der Intralogistik. Fraunhofer IAO, Stuttgart
Steinebach T, Wenzel J, Wakula J, Elbert R (2021) The human height as an input parameter for a capability-based order assignment in manual order picking. In: Arbeit HUMAINE gestalten, GfA Frühjahreskongress 2021, Bochum. GfA-Press, Dortmund

Stinson M, Wehking KH (2012) Leistungsbewertung und-optimierung in der manuellen Kommissionierung. Logist $\mathrm{J}$

Tompkins JA, White JA, Bozer YA, Tanchoco JMA (2010) Facilities planning. John Wiley \& Sons Inc., Hoboken

Ulin SS, Keyserling WM (2004) Case studies of ergonomic interventions in automotive parts distribution operations. J Occup Rehabil 14(4):307-326

Walch D, Galka S, Günthner WA (2009) Zwei auf einen Streich-Integrative Planung von Kommissionierprozessen durch die Kombination von MTM und der Leitmerkmalmethode

Waters TR, Putz-Anderson V, Garg A (1994) Applications manual for the revised NIOSH lifting equation, S 1-164 